\title{
Partners in academia and practice: Expediting and transforming scholarly nursing projects
}

\author{
Lynda J. Dimitroff ${ }^{* 1}$, Cheryl B. Crotser ${ }^{2}$, Donna M. Tydings ${ }^{3}$, Jeanine Seguin Santelli ${ }^{1}$, Laurie W. Ernest ${ }^{4}$, Lynn W. \\ Nichols ${ }^{5}$ \\ ${ }^{1}$ Nursing Department, Nazareth College, Rochester, NY, USA \\ ${ }^{2}$ Department of Nursing, Roberts Wesleyan College, Rochester, NY, USA \\ ${ }^{3}$ Department of Clinical Education \& Nursing Research Rochester Regional Health, Rochester, NY, USA \\ ${ }^{4}$ Department of Nursing Advanced Practice, Research \& Credentialing Highland Hospital, Rochester, NY, USA \\ ${ }^{5}$ Department of Nursing Research \& Evidence-Based Practice, Rochester Regional Health, Rochester, NY, USA
}

Received: January 24,2016

DOI: $10.5430 /$ jnep.v6n $7 \mathrm{p} 135$
Accepted: February 15, $2016 \quad$ Online Published: March 25, 2016

URL: http://dx.doi.org/10.5430/jnep.v6n7p135

\begin{abstract}
The introduction of evidenced-based practice and research, and the clinical application of knowledge is commonplace in accredited nursing curricula. Through projects, capstone development, and dissertations, students are provided opportunities to enhance knowledge and strengthen the pathways that lead to further professional query. It is essential that students practicing in the community are presented with standardized expectations and that they are uniformly mandated throughout the hosting clinical sites. A consortium that included nurse researchers from academic institutions and hospitals was formed to identify the elements of successful scholarly proposals. The goal of this project was to provide comprehensive and meaningful guidelines for the development of successful scholarly proposals. A packet was developed and used to guide students and health care organizations through the required steps for proposal approval. The change was implemented using Kotter's Eight Step Process for Leading Change.
\end{abstract}

Key Words: Collaboration, Standardized research expectations, Scholarly projects

\section{INTRODUCTION}

With the intent to establish a nursing research consortium, area nurse leaders held a meeting to discuss how nursing research conducted by students and faculty could be facilitated in the Rochester, NY region. The participants included nurse leaders from both academic and clinical settings. At this meeting, the idea of forming an innovative consortium between members of the local colleges and universities and their practice partners was entertained. The participants recognized that the university and clinical practice partners had unique knowledge and skills sets that could be shared and combined to strengthen the scholarly proposal process. Such collaborations promote excellence in nursing scholarship, education, and clinical practice. ${ }^{[1]}$

Participating in research is integral to advancing the nursing profession and clinical practice. It is necessary to achieve evidence-based care that will improve both nurse and patient outcomes. According to the American Nurses' Association (ANA) Code of Ethics "The nurse, in all roles and settings, advances the profession through research and scholarly inquiry, professional standards development, and the

*Correspondence: Lynda J. Dimitroff; Email: ljdimitr@aol.com; Address: 66 Hinsdale Street, Rochester, NY 14620, USA. 
generation of both nursing and health policy". [2]

The nursing community in the Finger Lakes Region has established a strong history of collaborative work. In 1997, the American Association of Colleges of Nursing (AACN) expressed their support for "... the concept that collaboration provides an environment for collegiality as well as mutual respect that includes shared planning, decision-making, problem-solving, goal-setting, responsibility, cooperation, and coordination". ${ }^{[1]}$ Additionally health care agencies and academic consortia allow for collaboration through partnerships. The partnerships encourage professionals to identify opportunities for improvement and advance the profession of nursing. ${ }^{[3]}$ Redding et al. ${ }^{[4]}$ suggested that the merging of collective information from multiple organizations yielded a product that resulted in substantial strength as opposed to the merging of few ideas from limited resources. Additionally historic information suggests that collaboration among broader groups more accurately reflects the needs of the whole, which in this case would be nursing.

The initial meeting resulted in the idea for an innovative collaborative to establish an academic-practice research alliance. Invitations were sent for an afternoon tea to members of the Rochester area nursing community to discuss research, collaboration, current resources, and the formation of a Finger Lakes Nursing Research Alliance (FLRA). At the conclusion of the Tea, attendees were invited to the inaugural meeting of the FLRA.

At the inaugural meeting, it was determined that the FLRA members were interested in supporting nursing scholarship through the facilitation of research and related activities such as evidence-based practice (EBP), nursing research (NR), performance improvement (PI), and program evaluation (PE). Engaging in these scholarly projects requires safeguards to assure: a) the ethical treatment and protection of human participants, b) confidentiality of protected health information, c) employee and patient safety, and, d) compliance with regulatory requirements and laws. As the first meeting unfolded, it was clear that each organization had a different process and format for nursing scholarly proposals. This process inconsistency was a barrier to the timely approval and conduct of nursing research and other scholarly work. The transformation of this process was completed by adapting the Kotter ${ }^{[5]}$ eight stage change process which included: 1) establishing the urgency for the need for change, 2) building a guiding team that is composed of leaders with the expertise and credibility to direct the needed change, 3) developing an effective vision and strategy to guide the transformation, 4) communicating the vision for change, 5) empowering action and removing barriers which includes training the customers,
6) creating short-term wins, 7) consolidating the gains, and 8) anchoring the changes in the process (see Table 1).

Table 1. The eight-stage process of creating major change

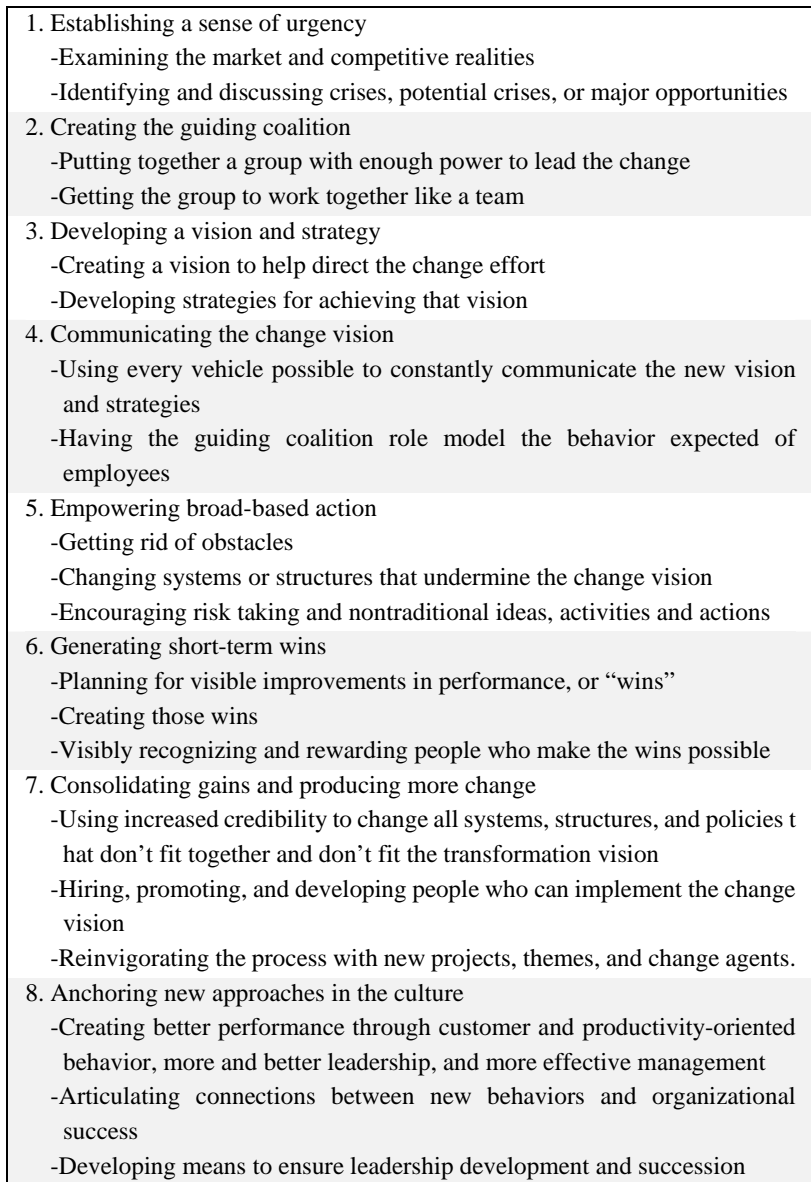

Source: John P. Kotter, Leading change, Harvard business review press. Reprinted with permission.

\section{Process}

\section{Stage One: Establishing the Need}

According to Kotter, ${ }^{[5]}$ the first step is to establish an urgent need for change. The sense of urgency was evident as graduate nursing programs required capstone or research projects to fulfill degree requirements. These projects necessitated approvals from practice partners at local hospitals. The practice partners were committed to supporting scholarly work, yet the process for submitting a request to conduct research or implement an evidence-based proposal varied among organizations. These inconsistencies resulted in project approval delays creating frustration for the academic faculty, students, and practice partners.

\section{Stage Two: Building a Team to Guide the Process}

A team of nursing research leaders in both the academic and practice settings guided the development of a standardized process. The leaders represented area colleges and health 
care systems. The stakeholders from both schools of nursing and practice partners who responded to the invitation to participate in the FLRA were eager for the opportunity to streamline the process for student and faculty research proposals and other scholarly activities. The members who actively participated were responsible for facilitating research in their member organization which created a guiding team with sufficient power to drive the change. The FLRA members became the guiding team and began to develop the FLRA mission, vision and values, and strategic plan, the third step of Kotter's ${ }^{[5]}$ process.

\section{Stage Three: Developing an Effective Vision and Strat- egy to Guide the Transformation}

The mission, vision, and values were created using unstructured brainstorming sessions to address (a) who we are, (b) why we exist, (c) who we serve, and, (d) what we wanted to accomplish. Once all the ideas were identified, the mission, vision and values were crafted collectively. The mission of the organization is to serve the Finger Lakes nursing research community and provide a collaborative support network of experts to facilitate the research process, the dissemination of research, and the translation of nursing research into practice. The vision of the organization is to be the premier regional resource for nursing research expertise. The group embraced the following values: innovation through curiosity, inquiry, collaboration, rigor, dissemination, evidence-based practice, and the timely translation of nursing research into practice. The initial project in the strategic plan was to formulate standardized process guidelines for submitting EBP, NR, PI, and PE projects, and white paper proposals.

\section{Stage Four: Communicating with the Community Mem- bers}

The FLRA members disseminated information to their respective institutions to increase awareness of their work and began gathering information on the current process used for the development of scholarly proposals. Each member took responsibility for developing draft guidelines and expectations for EBP, NR, PI, and PE projects, and white papers. An application checklist was developed to provide interested nurses with consistent requirements for completing scholarly work across facilities in the Finger Lakes region. Monthly meetings were held where consensus was sought and revisions were made until agreement on the standardized process was achieved.

Each checklist had a standard heading and signature page (see Table 2). Additionally, forms for administrative approval and site requests were developed. Consensus was achieved on each guideline with the caveat that there may be additional

Published by Sciedu Press requirements unique to individual organizations.

\section{Stage Five: Overcoming Barriers to Nursing Scholar- ship}

The checklists and application were developed to overcome barriers faced by nursing students, faculty members, and practice partners when conducting scholarly projects. A consistent process with clear expectations facilitates the research process for nurses by clarifying what must be submitted and the required approvals. With this standardized process, practice partners received completed applications facilitating a more timely approval of projects.

\section{Stage Six: Creating Short Term Wins}

Members of the Finger Lakes Research Alliance held a luncheon upon completion of the guidelines as a way to celebrate short term wins. Having created our documents, as identified above, the next step was to communicate the results of the project (the standardized proposal expectations) to all of the regional academic and practice partners who participate in research and other scholarly activities. The documents were created with input and representation from many of the area health care facilities and academic programs, however; not all stakeholders in the Finger Lakes region participated in the standardized process.

FLRA members identified the key messages to convey to the stakeholders and crafted talking points to guide the dissemination of this information. A packet including a cover letter, the FLRA Nursing Research/Project Site Request application, and the checklists (EBP, NR, PI, PE and white papers) was assembled and personally delivered to each regional organization where nursing research was conducted. Each guideline was also made available in an editable pdf format for ease in submission of research proposals. The FLRA brochure outlining the Alliance's membership, mission, vision and values accompanied the packet.

A cover letter was included in the packet to remind the stakeholder of the process and requests, how to access answers to questions, and to thank them for their time and participation. FLRA members then visited regional organizations where they discussed the talking points and left packets.

During the visit, several requests of the regional organization were made by the FLRA member. The first request was to identify a contact individual for students and researchers who wanted to conduct research within the facility. Secondly, they were asked to disseminate the information via their course syllabi or Nursing Research committees. And last, was a request for both the organization and the PI to complete an evaluation of the standardized application and checklists. 
Table 2. Nursing Research Checklist

Finger Lakes Research Alliance

Nursing Research Checklist

Title:

Applicant:_ Institutional Mentor:

Anticipated start date: __ Anticipated end date:

Student Project $\square$ No

$\square$ Yes If yes, Academic Advisor Name: Academic Institution:

\begin{tabular}{|c|c|}
\hline $\begin{array}{l}\text { Completed } \\
\text { or N/A }\end{array}$ & $\begin{array}{l}\text { This information must be included in your nursing research proposal. If you have a question about applicability of an item } \\
\text { to your study, contact academic or other institutional representative. }\end{array}$ \\
\hline & $\begin{array}{l}\text { Format Guidelines: } \\
\text { a. Cover page: Proposal title, researcher's name, credentials, proposal date } \\
\text { b. Lines of proposal must be numbered, beginning with cover page } \\
\text { c. Submit proposal in Microsoft Word. Do not submit a PDF file } \\
\text { d. Submit proposal and related appendices in one file. Multiple files will not be accepted } \\
\text { e. When revisions are submitted, assign a new date to the submitted version on cover page }\end{array}$ \\
\hline & $\begin{array}{l}\text { Abstract: Include the following sections: Background, Purpose, Research Question(s), Method, Results, Conclusions, Implications } \\
\text { for practice }\end{array}$ \\
\hline & Introduction \\
\hline & Research problem identification \\
\hline & Significance of studying the problem \& significance to nursing \\
\hline & Statement of purpose \& objectives \\
\hline & Research question(s) or hypothesis(es) \\
\hline & Theoretical (or conceptual) framework \\
\hline & Review of literature \\
\hline & Method and design \\
\hline & $\begin{array}{l}\text { Subjects/Participation/Sample \& Setting } \\
\text { a. Sample selection (inclusion and exclusion criteria) }\end{array}$ \\
\hline & b. Subject/Participant recruitment, including materials (if applicable) \\
\hline & c. Recruitment strategies \\
\hline & d. Participant compensation (if applicable) \\
\hline & e. Setting \\
\hline
\end{tabular}

** Forms available by contacting the primary author.

\section{Stage Seven: Consolidating Gains}

The Evaluation Plan for the project included two process evaluation forms, one for the PI and the other for the Chief Nursing Officer or research contact at the organization. The tools were constructed similarly for consistency. The focus of the tools was ease of understanding packet information, and comprehensiveness and relevance of information. Evaluators were asked to identify any other information that would have been helpful in the packet. Participants were also queried as to the likelihood of packet use in the future.

\section{Stage Eight: Anchoring the Changes}

The FLRA is continually striving to strengthen the culture of innovation and scholarship in the Rochester Nursing community. The FLRA continues to meet regularly with plans to evaluate the use of the packets. This will be accomplished through collation and discussion of the results from completed evaluations, maintenance of open communication with 
the stakeholders, and revising the packet based on the feedback received from academic and practice partners.

\section{Recommendations for Nurse Leaders}

Given that many of the area health care systems were either American Nurses Credentialing Center (ANCC) Magnet $($ recognized organizations or those seeking Magnet status, the information was well received. Standardizing the proposal process for research and other scholarly projects promotes improvement in care and the implementation of new knowledge to improve clinical practice.

Leaders in academic and practice settings have an important role in guiding and directing change by developing an inspiring vision and motivating others throughout the change process. An established change theory was used to create an environment for successful change with the ultimate goal of promoting nursing scholarship in the Rochester community.

Nevertheless, the change was difficult and many challenges were encountered. The members of the Alliance are leaders in both academic and practice organizations of the Rochester community and were fully supportive of the vision, mission and goals of the FLRA, yet competing priorities slowed the process down. One of the challenges identified since initiating this project is that the majority of area programs offering a master's of science degree have moved away from a research requirement to conduction of capstone EBP projects and proposals, or completion of comprehensive examinations. Additionally, turnover in the members of the Alliance was barriers to a smooth implementation of the standardized application and checklist. As with all projects, all the stakeholders have to be engaged. Cohen ${ }^{[6]}$ cautions that leaders of change must be persistent even after short term wins are realized to assure that the change becomes part of the established culture.

Use of Kotter's Eight Stage Change Process continues to be important as the FLRA evaluates the new process for scholarly project proposal submissions. Leaders have a key role in establishing the vision for changes, carefully plan- ning change implementation, and sustaining change efforts. The FLRA connected with the Deans and Directors of area schools and leaders of research and evidence-based practice in the clinical settings. It will be important to follow-through to assure that the information has been communicated and distributed to the faculty and staff working with students submitting scholarly project proposals for approval. Ideally, the change would be communicated to the entire faculty who supervise student projects.

\section{Conclusion/Discussion}

With this endeavor, the FLRA team recognized that collaborative projects can be challenging. They discovered very diverse individual characteristics and cultures embedded within each organization making standardization somewhat difficult to achieve. In addition, there were barriers, such as Institutional Review Board requirements, that were out of the team's control. Success at overcoming these barriers was achieved in part by engaging the appropriate stakeholders throughout the process. Doing so can be very helpful in the identification of problems and the development of workable solutions leading to successful collaborative projects. This change facilitates the conduct of research and evidence-based practice, ultimately serving the profession of nursing and our ethical responsibility to advance nursing science through scholarly activities. ${ }^{[2]}$

\section{ACKNOWLEDGEMENTS}

The authors of this article would like to recognize Dr. Cathryne A. Welch, former Director of the Cathryne A. Welch Center for Nursing Research. Dr. Welch founded the Research Alliances through the Foundation of New York State Nurses, and we thank her for her vision and assistance in the establishment of the Finger Lakes Research Alliance. We would also like to thank Drs. Charlene Smith and Pamela White for their work on this project.

\section{Conflicts of InTEREST Disclosure}

The authors declare that they have no competing interests.

\section{REFERENCES}

[1] American Association of College Nurses. Education and practice collaboration: Mandate for quality education, practice, and research for health care reform. J Prof Nurs. 1997; 13: 129-133. http://dx.doi.org/10.1016/S8755-7223(97)80013-0

[2] American Nurses Association. Code of ethics for nurses with interpretive statements. Silver Spring: Maryland: American Nurses Association; 2015.

[3] Frame KB, Ballantyne MJ, Haussler SC, et al. A collaborative model. Twenty healthcare agencies and academic institutions share resources to educate preceptors. Journal for Nurses in Staff Development. 2002;

Published by Sciedu Press
18: 185-193. PMid:12235417. http://dx.doi.org/10.1097/0 0124645-200207000-00003

[4] Redding DA, Anglin LT. How many is too many? Collaboration of multiple nursing organizations for professional development. The Journal of Continuing Education in Nursing. 2002; 33: 126-130. PMid:12046714.

[5] Kotter J. Leading change. Boston MA: Harvard Business Review Press; 2012.

[6] Cohen D. The heart of change field guide. Boston, MA: Harvard Business Review Press. 2005. 